in California have found that mutation, as well as deletion, of the gene changes how certain groups of neurons in the brain transmit signals. Signalling of a neuronal receptor that responds to cannabis and to endocannibinoids (which are made by the brain) is impaired in mice in which neuroligin-3 is mutated or missing. This suggests that disrupted endocannibinoid signalling contributes to autism, a mechanism that could suggest new strategies for treatment. Neuron http://dx.doi.org/10.1016/ j.neuron.2013.02.036 (2013)

\section{PALAEONTOLOGY}

\section{'Hobbit' brains not so small}

New estimates of brain size for Homo floresiensis make it more feasible that the diminutive hominid descended from Homo erectus.

The origins of $H$. floresiensis have been intensely debated in the decade since the roughly 18,000-year-old fossils of the 1-metre-tall hominid were discovered on the island of Flores in eastern Indonesia. Yousuke Kaifu at the University of Tokyo and his colleagues used replicas of an $H$. floresiensis skull and high-resolution computed-tomography scans to make models (pictured) of the hominid's brain. Their calculation of 426 cubic centimetres - roughly onethird the volume of a human brain, and the most accurate estimate so far - is slightly bigger than previous estimates. Just big enough, the authors say, that it is mechanistically possible that $H$. erectus underwent extreme dwarfism on an isolated island.

Proc. R. Soc. B 280, 20130338 (2013)

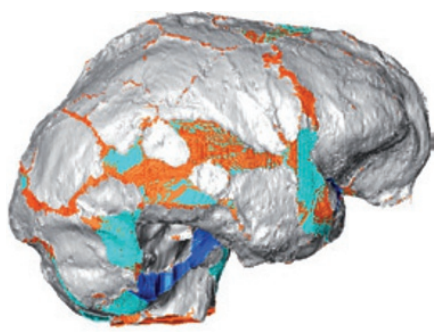

\section{Seeds travel on unpaved roads}

Dirt roads could be providing important corridors for seed distribution.

Alberto Suárez-Esteban and his colleagues at the Doñana Biological Station in Seville, Spain, collected animal faeces from 66 kilometres of manmade breaks in vegetation, such as firebreaks and dirt roads, as well as adjacent scrubland, in Doñana National Park in southwest Spain. The researchers identified and counted the seeds contained in 615 faecal samples from rabbits, carnivores and ungulates such as deer.

Carnivores and rabbits preferred to defecate on tracks, dispersing up to 124 times as many viable seeds along the tracks as in the scrub. Although ungulates avoided defecating along the tracks, their faeces also contained considerably fewer viable seeds.

The authors suggest that such human disruptions could have an overlooked role in plant conservation by helping animals to spread seeds between isolated plant populations, but they could also provide routes for invading species.

J. Appl. Ecol. http://dx.doi. org/10.1111/1365-2664.12080 (2013)

\section{CLIMATE CHANGE}

\section{Evolution in acidic oceans}

An increase in ocean acidity could drive substantial genetic change in sea urchins in just one generation.

Melissa Pespeni at Hopkins Marine Station in Pacific Grove, California, and her colleagues housed developing purple sea urchins (Strongylocentrotus purpuratus) under current acidity levels and the higher levels that are expected from increasing amounts of carbon dioxide in the

COMMUNITY CHOICE

The most viewed papers in science

\title{
Nanospheres make clever membranes
}

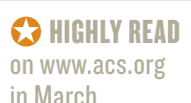

in March

Spheres of silica coated in gold have been made into membranes whose permeability can be engineered.

Ilya Zharov and Patricia Ignacio-de Leon at the University of Utah in Salt Lake City created nanospheres that self-assemble into arrays, and can then be heated to make inorganic membranes. By coating the silica spheres with gold, the duo were able to attach a variety of chemical groups to the spheres. Surface modifications affected how various molecules passed through the membranes, a process that could be further controlled by changes in $\mathrm{pH}$. Such materials could have applications in chemical separations, catalysts and sensors, the authors say. Langmuir 29, 3749-3756 (2013)

atmosphere. The authors measured changes in the frequency of 19,493 genetic variants as fertilized eggs grew into swimming and feeding larvae. Although conditions of high acidity had little effect on the growth of the animals, major shifts occurred in genes that code for 40 classes of proteins. These changes were concentrated in genes related to the construction of urchins' shells and how the organisms regulate metabolism and $\mathrm{pH}$.

Increased acidity could be selecting for genetic variants that improve survival under these conditions, the researchers suggest.

Proc. Natl Acad. Sci. USA http://dx.doi.org/10.1073/ pnas.1220673110 (2013)

\section{Dusty galaxies come into view}

Astronomers have made their first statistically reliable survey of one kind of star-forming galaxy in the early Universe.

Knowledge of these distant objects is important for our understanding of these galaxies' formation and evolution, but enshrouding dust usually obscures their

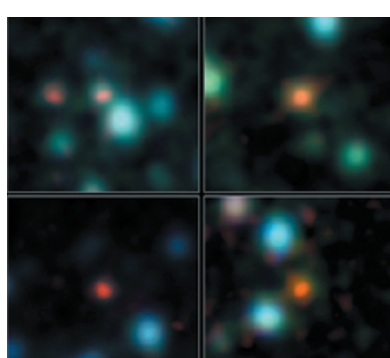

details - making them hard to identify with telescopes that collect radio waves or visible light. Jacqueline Hodge at the Max Planck Institute for Astronomy in Heidelberg, Germany, and her colleagues used the Atacama Large Millimeter/submillimeter Array (ALMA) in Chile to penetrate the dust veil by looking for emissions at submillimetre wavelengths of light - a length between infrared and radio waves.

The scientists' observation of 126 previously unresolved galaxies in the southern constellation Fornax brought blurry objects into sharper focus (pictured). At least one-third, and possibly up to one-half, of them turned out to be multiple galaxies.

Astrophys. J. 768, 91 (2013)

\section{$\rightarrow$ NATURE.COM}

For the latest research published by Naturevisit:

www.nature.com/latestresearch 